\title{
PHARMACODYNAMIC SUBEPIDERMAL TESTS: II, INDIRECT TESTS; A, THYROIDIN
}

\author{
M. Ascoli and A. Fagiuoli \\ (From the Institute of Internal Medicine, University of Catania, \\ Director, Prof. M. Ascoli) ${ }^{1}$
}

A series of investigations is being undertaken in our Institute in an endeavor to ascertain to what extent the intracutaneous injections of endocrin substances and those especially related to the autonomic nervous system can be utilized in practical diagnosis. In a previous issue of this Journal, the results secured with pituitrin were briefly described (2).

The desirability of investigating thyroid material in this way was obvious although it seemed, a priori, less promising on account of our inability to utilize a definitely identified, pure active principle.

As compared with a control subepidermal injection of water, that of thyroid extract differs in giving rise to a larger papule and a greater degree and duration of erythema. The reaction was found to vary a great deal with the individual, however, being now more active and lasting, now so attenuated as to become indistinguishable from the control reaction. But the same variability in the reaction picture is seen following injections made in a given subject at the same time with other extracts, e. g., of ovary, thymus or testicle. The problem does not seem to lend itself, therefore, to a simple, direct attack. Can it be solved by indirect methods?

Among the different endocrin organs there are close relationships of interdependence, synergy or antagonism. In the case of adrenalin and pituitrin, characteristic reactions are directly attainable by means of subepidermal injections. In case of other endocrin organ extracts, as above mentioned, unequivocal reactions are not readily secured. The problem then presents itself : Can the adrenalin reaction be characteristically alteredattenuated or accentuated-by the use of extracts of different endocrin organs? The sensitivity of the organism to such materials might thus be disclosed. As a case in point, does the addition of thyroid extract in a quantity not in itself causing reaction lead to augmentation or attenuation of the adrenalin cutaneous re- 
action as it has been reported by Falta to affect the metabolic reaction and by Oswald and others to affect the vasomotor reaction?

The test has given positive results in certain pathological but not in normal subjects (3). A given individual reacts distinctly to adrenalin in $1: 200,000$ dilution, but only feebly to $1: 1,000,000$. If to the adrenalin in these dilutions a small quantity of thyroid extract ( 0.01 to 0.005 ce.) be added (4) - a quantity which in itself gives no direct reaction distinguishable from that to distilled water,--the adrenalin reaction is distinctly enhanced and more protracted, as compared with the reaction to the adrenalin alone. The central red color in the reacting zone is also often deeper.

The same activation may be demonstrated in case of pituitrin instead of adrenalin and this second indirect test may be found in cases in which the former is negative (5).

Contrary to the results obtained with thyroid extract, other endocrin glands seem to attenuate the adrenalin reaction. We shall have occasion to report further upon these latter extracts as well as upon the clinical significance of the indirect thyroidin test. 1920.

1. Submitted to the Reale Accademia dei Lincei, Rome, March,

2. Ascoli, M. and Fagiuoli, A.: Pituitrin test. This Journal, 1920, 4, 33-36.

3. It is presumed that the failure to obtain the phenomenon in the normal subject is due to the insufficient amount of active material in the thyroid extract employed. The result was the same, however, with Oswald's thyreoglobulin.

4. The extracts employed by us are of 25 per cent strength in physiological saline solution, obtained from bovine thyroids under a pressure of $350-400 \mathrm{~atm}$. to the sq. $\mathrm{cm}$. The extract is filtered through porcelain, preserved with $1 / 2$ per cent chloretone and distributed in sterilized vials. For this preparation we are indebted to the "Instituo nazionale medico farmacologico" of Rome.

5. We have frequently alluded to the necessity of making the injections superficially and strictly subepidermically. This condition is all the more essential in the thyroidin test in whch are to be compared only differences in intensity of reactions of the same category such as could readily be produced merely by variations in the depth of the injections. In order to obviate error when differences are small, the test may be made in duplicate or triplicate and be regarded as positive only when the result is obvious and the difference easily appreciable to one not concerned in the result. 